\title{
HIGHER POLE LINEAR TRAPS FOR ATOMIC CLOCK APPLICATIONS
}

\author{
JOHN D. PRESTAGE, ROBERT L. TJOELKER, AND LUTE MALEKI \\ , \\ Jet Propulsion Laboratory, California Institute of Technology, 4800 Oak Grove Drive, Mail Stop 298-100 \\ Pasadena, CA 91109
}

\begin{abstract}
We investigate experimentally and theoretically higher pole linear ion traps for frequency standard use. We have built a 12-pole trap and have successfully loaded ions into it from a linear quadrupole trap. By solving the Boltzmann equation describing large ion clouds where space charge interactions are important, we show that clock frequency changes due to ion number fluctuations are much smaller in ion clocks based multipole traps than comparable clocks based on quadrupole linear traps.
\end{abstract}

\section{INTRODUCTION}

To date all linear ion traps used for atomic clock applications have been quadrupolar in their $\mathrm{rf}$ confining electric field pattem. The pseudopotentials for such trapping fields are equivalent to a uniform background pseudo-charge with a consequence that (cold) ion clouds fill the trap with uniform density. Large ion clouds in linear. traps have shown excellent short-term frequency, $\sigma_{y}(\tau)=3 \times 10^{-14} / \sqrt{\tau}$, but a large second-order Doppler shift can lead to frequency instabilities. Most of this second order Doppler shift is a consequence of the space charge Coulomb repulsion force that results from confining ions of like charge in a relatively small volume. These repulsive forces are balanced by the ponderomotive forces generated by ion motion in the $f$ electric field gradient. To achieve a good signalto-noise ratio in the measured clock resonance in a lamp based clock, large ion clouds of $\sim 10^{7}$ ions in a $65 \mathrm{~mm}$ long cloud are required. Most of the motional energy in the cloud is thus stored in the micro-motion to generate the force to balance the space charge. For a typical cloud, buffer gas cooled to $500 \mathrm{~K}$, the Doppler shif from if mictomotion will be about 3 times the secular motion contribution to the energy. By contrast, the 12pole will hold the same number of ions with only $1 / 3$ of the motional energy stored in the micro- motion, nearly 10 times less than the quadrupole trap.

In this paper we examine the use of a 12-pole linear trap for clock applications. The pseudopotential well and the if confining fields are dramatically smaller in the trap interior for higher pole traps and grow rapidly near the trap electrodes. In the absence of space charge interactions, ions can spend most of their trajectory in the field free interior of the trap.

\section{EXPERIMENTAL APPARATUS}

We have developed a 12-pole ion trap to investigate the experimental challenges of operating a multi-pole trap for $\mathrm{Hg}$ ions as an atomic clock. The 12-pole trap is essentially closed allowing no optical interrogation of the trapped $\mathrm{Hg}$ ions within. To carry out optical preparation and interrogation, the ions are created in a more open quadrupole trap where their fluorescence can be monitored. They are electrically shuttled into the 12-pole trap and

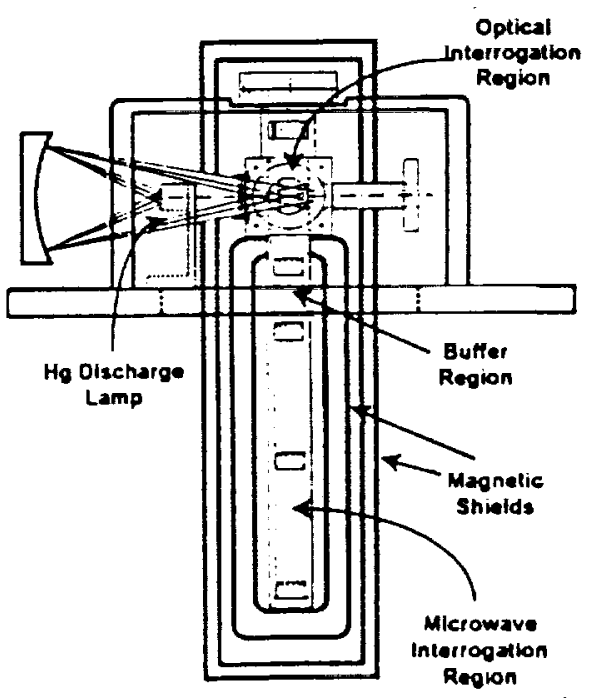

Figure 1. 
shuttled back to the quadrupole trap to optically measure any state changes that have been made in the clock transition. The apparatus is shown in Figure 1

In Figure 1, the optical interrogation region " houses a linear quadrupole trap where $194 \mathrm{~nm}$ light from a ${ }^{202} \mathrm{Hg}$ discharge lamp is focussed onto the ion cloud with fluorescence being collected in a direction normal to the page. An electron gun injects a beam of electrons down the axis of the trap ionizing a neutral vapor of ${ }^{199} \mathrm{Hg}$ atoms at a pressure $\sim 10^{-9} \mathrm{Torr}$. A helium buffer gas $\left(\sim 10^{-5}\right.$ Torr) cools the ions to $500 \mathrm{~K}$ or so and greatly enhances the number of trapped ions.

- The quadrupole trap is along the same axis as the 12-pole trap which begins just below the optical cube and extends into the microwave interrogation region to the bottom of the vacuum tube. The cylindrical vacuum tubing below the optical cube is wound with a 2 turn solenoid over the full length of the 12-pole trap, extending several centimeters beyond the 12-pole trap at each end. A small dielectric filled waveguide brings $40.5 \mathrm{GHz}$ microwaves from the upper access flange into the lower mictowave interrogation region.

Ions are shuttled from the quadrupole trap into the 12-pole trap by applying a positive dc bias to all 4 rods of the quadrupole relative to the 12 rods of the multi-pole trap. [1]

\section{LARGE ION CLOUDS IN MULTI-POLE TRAPS}

Higher pole traps are used to study ion-molecule low energy collisions and reactions [2]. Octopole if electrodes act as a guide to transport ions from one location to another in similar applications. These applications involve very low densities of ions where space charge interactions within the ion cloud are inconsequential. Ions are detected directly with a channeltron electron multiplier. Because the ions in a multi-pole if trap can spend very little time in the region of high rf electric fields there is very little rf heating and low temperature collisions can be studied. In this section we develop the theory of large ion clouds in multipole traps by solving the Boltzman equation which will give the spatial distribution of ions at temperature $T$ held in the pseudo-potential well of the multi-pole trap. This will be a two dimensional density profile appropriate to a long linear trap configuration where fringing fields near the ends are much shorter than the total trap length.
The pseudo-potential, $V$, for a particle of charge $q$ and mass $m$, inside the linear multi-pole is given by [2]

$$
V^{*}(r)=\frac{k^{2}}{16} \frac{q^{2} V_{0}^{2}}{m \Omega^{2} r_{0}^{2}} \hat{r}^{2 k-2}
$$

The number of electrodes comprising the trap is $2 k$, the operating if frequency is $\Omega$ radians per second, the trap inner radius is $r_{a}$ and the amplitude of the if voltage applied to each rod is $V_{0} / 2$. (The peak voltage between neighboring trap electrodes is $V_{a}$ ) The radial coordinate, $r$, is measured outward from the trap centerline with the normalized distance

$$
\hat{r}=\frac{r}{r_{0}} .
$$

The radial density , $n(r)$, of ions at temperature, $T$, held in the multipole trap is found from the Boltzmann distribution $[3,4]$

$$
n(r)=n(0) \exp \left\{\frac{-V^{*}(r)-q \varphi_{x}(r)}{k_{B} T}\right\}
$$

Using Poisson's equation for the space charge potential from the ion cloud of density $n(r)$

$$
\nabla^{2} \varphi_{s c}(r)=-q n(r) / \varepsilon_{0}
$$

We find that $n(r)$ satisfies the second order nonlinear equation

$$
n^{\prime \prime}+\frac{n^{\prime}}{r}-\frac{n^{\prime 2}}{n}-\frac{n^{2}}{n_{0} \lambda_{D}^{2}}=-k^{2}(k-1)^{2} \frac{n}{4 \lambda_{D}{ }^{2}} \hat{r}^{2 k-4}
$$

In this equation, $n_{0}=2 \varepsilon_{0} \mathrm{mos}^{2} / \mathrm{q}^{2}$ is the pseudocharge density of a quadrupole trap with secular frequency $\omega$, and $\lambda_{D}^{2}=k_{B} T /\left(2 m \omega^{2}\right)$ is the Debye length for the ion plasma.

To compute the second-order Doppler shift for the ion cloud density distribution we must also know the micro-motion velocity throughout the ion trap. This is given by

$$
\left\langle v^{2}\right\rangle=\frac{k^{2}}{4} \omega^{2} r_{0}^{2} \hat{r}^{2 k-2}
$$


And can be averaged over the density distribution to obtain the mean velocity stored in the micro-motion. Notice that for higher pole traps the micro-motion is small over the interior of the trap.

We solve the Boltzmann equation for the density profile by a fourth-order Runge-Kutta numerical technique. Typical operating conditions are 70 volts peak voltage between neighboring rods with a $1 \mathrm{MHz}$ drive frequency. Assuming the $\mathrm{Hg}$ ions are at $700 \mathrm{~K}$ temperature we find a solution to the Boltzmann equation as shown in Figure 2 below. The density profile has been normalized to $n_{0}=2 \varepsilon_{0} \mathrm{mos}^{3} / q^{2}$ as previously defined.

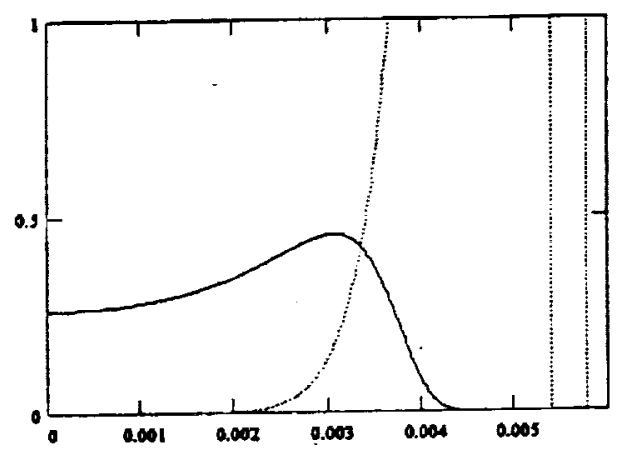

Figure 2.Normalized ion density inside a 12-pole trap of inside radius $0.0056 \mathrm{~m}$. Ion temperature is $700 \mathrm{~K}$.

This density corresponds to $1.5 \times 10^{7}$ ions in a 25 $\mathrm{cm}$ long 12-pole trap. In Figure 2 we have also plotted the normalized second order Doppler shift from the two dimensional micro-motion vs radial position. This is defined as follows

$$
\frac{-\left\langle\frac{v^{2}}{2 c^{2}}\right\rangle}{-\frac{k_{B} T}{m c^{2}}}=\frac{k^{2}}{8} \frac{m \omega^{2} r_{0}^{2}}{k_{B} T} \hat{r}^{2 k-2} \equiv F_{d}^{k}(r)
$$

Notice that the normalized $2^{\text {nd }}$ order Doppler from the micto-motion is less than the ion thermal motion throughout most of the interior of the cloud.

Finally, we compute the total $2^{\text {nd }}$ order Doppler from the micto-motion averaged across the ion cloud as

$$
N_{d}^{k}=\frac{\int n(r) r F_{d}^{k}(r) d r}{\int n(r) r d r}
$$

From this calculation we find the total second order Doppler shift for the trapped ions with density profile $n(r)$

$$
\frac{\Delta f}{f}=-\frac{3 k_{B} T}{2 m c^{2}}\left(1+\frac{2}{3} N_{d}^{k}\right)
$$

We can now evaluate this expression numerically for any multi-pole linear trap with a given total number of ions per unit length and temperature.

We first note that in the limit of small ion clouds where space charge effects are negligible,

$$
N_{d}^{k}=\frac{1}{k-1} \text {. }
$$

Thus, in the linear quadrupole trap with only a small number of non-interacting ions (space charge effects are negligible) $N_{d}=1$. This is a consequence of the equality of the average secular energy and average micro-motion energy in a harmonic (quadrupole) trap. As space charge interaction grows larger, $N_{d}=N_{d}^{k=2}$ increases. This number is typically 3 for buffer gas cooled $\mathrm{Hg}$ ion clocks.

The 12-pole, by contrast, begins in the small cloud limit as $N_{d}^{k=\sigma}=1 / 5$, already 5 times smaller than the quadrupole. Additionally, because the field-free interior volume of the multipole is much larger than the quadrupole with the same radius $R$, the low density limit is satisfied with a much larger number of ions. This is illustrated in Figure 3 where the total $2^{\text {nd }}$ Doppler vs ion number is shown for the $65 \mathrm{~mm}$ quadrupole we have used for $\mathrm{Hg}$ clock work and the $25 \mathrm{~cm}$ long 12-pole trap currently under development. In both traps the ions are at $700 \mathrm{~K}$ as the number is varied. The point to emphasize here is that changes in ion number in the 12-pole trap influence the total $2^{\text {nd }}$ Doppler shift more than 10 times less than the same change in the quadrupole trap

\section{FIRST RESULTS IN A 12-POLE TRAP}

We have built a 12-pole trap in the configuration shown in Figure $I$. lons have been successfully 


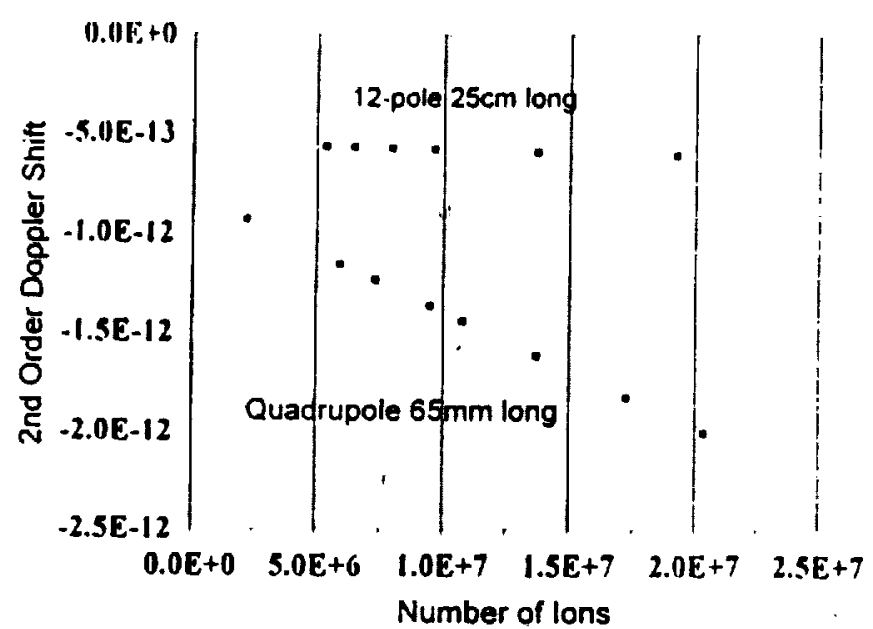

loaded into the quadrupole trap where the atomic fluorescence was measured with good signal to noise. The ions were then electrically shuttled into the co-linear 12-pole trap. They were then

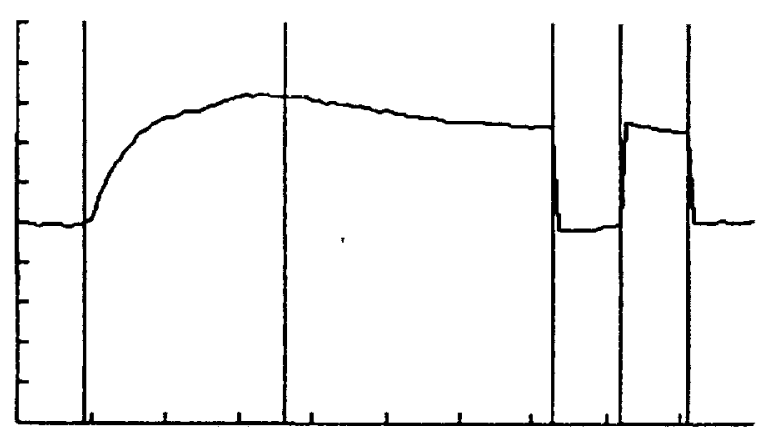

Figure 4

shuttled back into the quadrupole where fluorescence measurements show that none of the ions were lost in transport across the junction between the two traps. This also demonstrates that none were lost from the 12 pole trap. This is important because the well depth of the 12-pole is around $0.3 \mathrm{eV}$ as compared to the $3+\mathrm{eV}$ well depth of the linear quadrupole trap.

The fluorescence sequence as the ions are loaded into the quadrupole trap and then moved into the 12-pole trap and then back to the quadrupole is shown in Figure 4.

\section{SUMMARY}

We are investigating the use of higher pole traps for $\mathrm{Hg}^{+}$ion frequency standards. We have built a 12-pole trap and have shutued ions into it from a linear quadrupole trap. After many seconds trapped in the 12-pole trap, ions are shuttled back into the quadrupole, demonstrating trapping of large ion clouds where space charge interactions are non-negligible in a multi-pole trap. We have solved the Boltzmann equation describing large ion clouds in the general multipole trap of arbitrary order. We have shown that fluctuations of the number of trapped ions influence the clock frequency much less severely than in the quadrupole case.

This work was performed by the Jet Propulsion Laboratory, Califomia Institute of Technology, under contract to the National Aeronautics and Space Administration.

\section{REFERENCES}

[1] J. D. Prestage, R. L. Tjoelker, G. J. Dick, and L. Maleki, "Progress Report on the Improved Linear Ion Trap Physics Package," pp. 82-85, 1995 IEEE International Frequency Control Symposium, San Francisco, USA, 1995.

[2] D. Gerlich, "Inhomogeneous RF Fields: A Versatile Tool for the Study of Processes of Slow Ions," Adv. In Chem. Phys. LXXXII, pp. L-178, 1992.

[3] L. S. Cutler, C. A. Flory, R. P. Giffard, and M. D. McGuire, "Doppler Effects due to Thermal Macromotion of lons in and of Quadrupole Trap," Applied Physics B 39, pp. 251-259, 1986.

[H] J. D. Prestage, R. L. Tjoelker, G. J. Dick, and L. Maleki, "Doppler Sideband Spectra for lons in a Linear Trap," pp. 148-154, 1993 IEEE Intemational Frequency Control Symposium. Salt Lake City, USA. 